\title{
Post-Publication Peer Review for Real
}

\author{
Koki Ikeda, Meiji Gakuin University and Keio University \\ Yuki Yamada, Kyushu University \\ Kohske Takahashi, Chukyo University
}

\begin{abstract}
The inefficiency of the current peer-review system has been discussed for many years, and now there is a surge of various countermeasures aiming to solve the problems. Post-publication peer review (PPPR) has emerged as one of them, and some scholars expected that it would be the definite solution. Unfortunately, a decade of trial has not turned out to be as fruitful as expected. We assessed that the biggest reason for this situation was the lack of incentives among contributors, and proposed that publishing review commentaries as independent and qualified publications in a dedicated section of a journal might solve the problem. Specifically, we took the open peer commentary section of Behavioral and Brain Sciences as a model of such an incentivised structure, and pictured a possible implementation of this idea in the current web-based environment. Potentials of this new PPPR format were suggested. (143 words)
\end{abstract}


A recent large-scale survey reported that $98 \%$ of researchers who participated in the study agreed that the peer-review system was important (or extremely important) to ensure the quality and integrity of science. In addition, $78.8 \%$ answered that they were satisfied (or very satisfied) with the current review system (Publon, 2018). It is probably true that peer-review has been playing a significant role to control the quality of academic papers (Armstrong, 1997). The latter result, however, is rather perplexing, since it has been well known that sometimes articles could pass through the system without their flaws being revealed (Hopewell et al., 2014), results could not be reproduced reliably (e.g. Open Science Collaboration, 2015), decisions were said to be no better than a dice roll (Lindsey, 1988; Neff \& Olden, 2006), and inter-reviewer agreement was estimated to be very low (Bornmann et al., 2010). Thus, the reported high satisfaction for the status quo suggests that we might be either simply blind or unwilling to admit that there is a huge room for improvement (but see, Ross-Hellauer et al., 2017; Taylor \& Francis Group, 2015).

The low reliability across reviewers is especially disturbing and raises serious concerns about the effectiveness of the system, because we now have empirical data showing that inter-rater agreement and precision could be very high, and they robustly predict the replicability of previous studies, when the information about others' predictions are shared among predictors (Botvinik-Nezer et al., 2020; Camerer et al., 2016, 2018; Dreber et al., 2015; Forsell et al., 2019). Thus, the secretiveness of the current system could be the unintended culprit of its suboptimality.

\section{PPPR}

One of the proposed solutions for this problem is post-publication peer review (PPPR, henceforth; Amsen, 2014; Brown, 2016; Eisen, 2016; Knoepfler, 2015; Kriegeskorte, 2009; Sullivan, 2018; Tennant, 2017). Here, we refer to PPPR as a review system that is open for general scholarly volunteers (or in some cases particular people who are 
assigned by the original authors) to critically evaluate an academic paper, which could be either reviewed already or not, after its public appearance. In addition to the general benefit of openness, Andrew Gelman, for example, argued that PPPR was the most efficient way to avoid the problems of the current review system. He maintained that PPPR could rapidly mobilize the potential reviewers outside the original secretive review process, in response to the information about the possible academic and/or social impact that the target article could have, and therefore could prevent its potentially negative influence spreading all over the academia as well as media outlets (Gelman, 2016).

Gelman's idea of massive and efficient mobilization of reviewers is appealing especially when it is combined with the successful records of the replication market reported so far. Let us imagine the following scenario. A paper which contains some controversial aspects is published in a journal and causes a stir. Some PPPR systems quickly recruit quite a few number of reviewers, and their commentaries are publicly uploaded and therefore the audience can see how others evaluate the target study. Then, some of them write follow-up reviews and post them again as PPPR, which creates a positive feedback loop. Eventually, this cycle leads to a precise and shared assessment of the study in a short time. In fact, this is not entirely fictional. Similar events happened in some cases of scientific fraud (e.g. Meskus et al., 2018), having proved that PPPR could be far more effective than the current review system. Then, why aren't we using it?

\section{Problems}

More than a few PPPR systems are working currently. Examples are PubPeer (https://pubpeer.com/), Hypothes.is (https://web.hypothes.is/), e-Letters (https://www.sciencemag.org/help/e-letters), bioRxiv (https://www.biorxiv.org/), ScienceOpen 
(https://f1000research.com/) among others. Obviously, the main goal of these projects is to liberate the current review system from its secrecy and ineffectiveness in order to change the system drastically in a better direction. It is, however, probably fair to say that they have not become as popular as originally intended. In fact, some major journals and literature databases had tried to establish their versions of PPPR, but only ended up in vain, mainly because of their unpopularity (Dolgin, 2018; McCook, 2018; Nielsen, 2009). The same trend can be found in a survey that asked how comfortable researchers felt with the idea of PPPR (Taylor \& Francis Group, 2015). The answer was in the range between 4.4 and 5.7 on the 10 point scale with 10 as very comfortable (the actual scale was asking it in an agree-disagree style), suggesting they were not strongly motivated to support it.

Perhaps the biggest obstacle is its weak incentive structure. This problem is not limited to PPPR and the current review system also greatly suffers from it. In fact, it was reported that $84.8 \%$ of researchers in a survey answered that their research institutions/universities should have incentivized and recognized more about their review activities, and $83.3 \%$ of researchers said that a greater recognition and career incentives would create a positive (or extremely positive) impact on the efficacy of the process (Publon, 2018). The same report also revealed that the main reasons they accepted the review invitation were either that they thought it was a part of their job, or they must have followed the social norm of reciprocity. Apparently, writing a review was perceived as a civil obligation, not reward.

Presumably, these are natural consequences of the current mainstream academic culture. Review commentaries are not treated as publications and therefore cannot be enlisted in one's CV. Financial compensation is probably not a realistic solution given the lack of abundant resources. Since writing a precise and thoughtful commentary requires a lot of time and effort, these conditions can strongly discourage the potential reviewers. Publon (https://publons.com/) has launched its service with a goal to reverse 
such a trend by making the review process rewarding (Johnston, 2015). Although it is definitely too early to judge the outcome of this project, a recent survey showed that the willingness to accept review invitations had been unchanged or possibly declined (Publon, 2018) as compared to the previous reports (Bravo et al., 2019; Taylor \& Francis Group, 2015). There seems to be no turn of the tide yet. Although we are not aware of similar surveys focusing on PPPR, it is reasonable to assume that the situation is the same, or possibly worse given its unfavorable reputation.

Another reason for the unpopularity of PPPR could be its low readability. Twitter is the most extreme example of this problem. Fast exchanges of analyses and commentaries sometimes make it an ideal medium to foster valuable post-publication discussions. However, it has many drawbacks too. Arguments can be scattered over ever-branching threads and sometimes it could be very hard to find even who started the discussion originally. Relevant information such as hyperlinks to papers, uploaded data, analysis codes, and blog posts etc. is so fragmented that one has to open a countless number of browser pages, and go back and forth between them. The same kind of problem can be found in the threads of short commentaries on the current PPPR system, maybe to a lesser extent though. Moreover, since there are currently so many different platforms for PPPR (Tennant, 2017; Van Noorden, 2014), researchers have to search all of them if they want to catch all relevant information. In summary, it is clear that a better platform for PPPR is needed in regards to not only the motivational but also implementational aspects.

\section{A Model}

Fortunately, there has already been an interesting and unique attempt for the PPPR system in psychology that might provide a solution for these problems; that is, the open peer commentary section in Behavioral and Brain Sciences (BBS, henceforth; https://www.cambridge.org/core/journals/behavioral-and-brain-sciences). We will firstly 
look into some distinct features of this system in this section, and then take it as a model for developing a blueprint for a better PPPR system in the following sections.

Some characteristics are especially notable for BBS commentary. Admittedly, to pair a target article with several commentaries is not a rare editorial activity. You can find it in, for example, Psychological Inquiry and other special themed issues in various journals. However, BBS commentary is different from them in terms of that it employs an "open" system so that anyone can submit the commentary proposal at will (although some commenters are arbitrarily chosen by the editor). This characteristic makes BBS commentary much more similar to PPPR than other traditional publications.

Next and most importantly, the majority of researchers find no problem with their incentives to submit an article as a BBS commentary, because they will be considered by many researchers and institutes to be equivalent to a genuine publication and can be listed on one's CV. Therefore, researchers have strong incentives to actively publish their reviews on BBS. There seem to be at least two reasons for this uniqueness. Firstly, BBS is undoubtedly one of the most prestigious journals in psychology and its related areas, with a 17.194 impact factor for the year 2018. Secondly, the commentaries are selected by the editor before publication, so their quality is guaranteed at least to some extent. Critically, no current PPPR has the features comparable to these in BBS.

Finally, although this could be a matter of subjectivity to some degree, the readability of $B B S$ seems to be relatively high for the majority of the audience, as compared to the thread-style, simple succession of short commentaries generally found in the current PPPR system. In BBS, each commentary is well paraphrased and organized, quite contrary to a tweet-like short scribble. Readers can continue reading each commentary one by one until they reach the original authors' response at the end of the segment. They are not interfered by the insertions of pros and cons from other commenters, 
which sometimes occupy most of the discussions in other PPPRs. This high readability is merely a result of the fact that BBS simply follows the traditional journal design, but still it serves well to the audience.

\section{Adjustments}

Thus, the open peer commentary system in $B B S$ seems to be a promising model for a better designed PPPR. However, of course there are some rooms for adjustment and improvement. First and foremost, the target articles in BBS are limited to those that have already been peer-reviewed and will be published in the same journal. In contrast, the fundamental idea of PPPR is that any paper appeared in the public should be a potential target of the review process. PPPR should not be a property of some special journals, but a common good for the entire academia. It is obvious that a flexible and efficient allocation of human resources to relevant papers across journals cannot be achieved without such openness (Gelman, 2016).

Second, the number of commentaries is also limited in $B B S$ due to its periodic publication, and this would nullify the core function of PPPR and therefore must be changed. In PPPR, the review process is an open-ended system, where the number of commentaries could be ever-increasing into the future. We cannot predict when the evaluation of a paper, which we thought was once established, suddenly or gradually capsizes in the face of new empirical data (e.g. Doyen et al., 2012; Hagger et al., 2016; Wagenmakers et al., 2016), new analysis (e.g. Rohrer et al., 2017; van der Zee et al., 2017), excavation of historical facts (e.g. Le Texier, 2019; Perry, 2012), and even whistleblowing (e.g. Jha, 2012). Rapid checks and adjustments of the evaluation must require no limit for the number of commentaries on the target article.

In fact, due to the recent rapid technical innovations, these features have already been fully implemented in many PPPRs currently working, such as PubPeer and Hypothes.is. 
The widespread use of prepreints, such as bioRixv, PsyArXiv (https://psyarxiv.com/), and others has also promoted the public characteristics (open to any papers) of PPPRs. The number of commentaries also has no apparent limit (open to any commentaries) in any of these services.

To summarize, we might be able to create a new and better PPPR system by simply combining the advantages of BBS commentary - (1) strong incentive for commenters and (2) high readability - with those of the current PPPRs - (3) unlimited target selection and (4) unlimited commentary accumulation -. In the next section, we propose a possible blueprint for the implementation of these ideas, especially with a focus on the first two, because the rest has already been realized in the current media.

\section{A Blueprint}

(1) Incentive: launch a new section dedicated to the publication of PPPR in a journal with high transparency

Among the features that seem critical to the successful implementation of PPPR, strong incentives for commenters is probably the most important factor. We speculated that $B B S$ has achieved this goal by providing the commentaries a status equivalent to a standard academic paper. Furthermore, this is probably realized by the journal's two unique characteristics: its academic prestige and the selection of commentaries by the editor. Based on these considerations, we propose the following plans for the new PPPR system.

Firstly, commentaries must be "published" in a way similar to standard academic papers. Of course this does not mean that it has to be literally printed in a physical material. Instead, the publication of each commentary should be officially announced and recorded with a specific DOI on the journal's website as well as public literature 
databases, and therefore authorized by the editorial board. Moreover, such a demarcation should be accompanied by a minimal screening process by a (or a team of) journal editor(s) regarding the tone and appropriateness of the commentary, possible conflict of interests and other conceivable problems (cf. Brown, 2016; Eisen, 2016). These processes can be contrasted with the majority of the current PPPR, which simply and automatically add new commentaries without any conspicuous engagement from the editorial team. Such a policy might leave an impression to the audience that the commentary section looks more like a lawless battlefield rather than a place for proper academic exchanges, even though effort for curation is in fact being made behind the scene in many PPPRs (Neuroskeptic, 2015; Tennant, 2017). An official publicization of commentaries by the journal could give a sense of trust for the audience and potential contributors. It is no doubt that these activities will add extra work for editors, but we believe it is worth doing because it could revolutionize the efficiency of the review system as a whole.

Secondly, the selection process for the review commentaries must be open to the public too. Although it is supposed to be minimal (i.e. not a fully-implemented review), any secrecy during the manuscript handling could impair the trust among the potential contributors. We propose a style similar to the revision history pages of Wikipedia (https://www.wikipedia.org/) for this disclosure. In this format, the audience who are interested only in the editor-selected commentaries can read through them without any distractions, as in the main pages of Wikipedia. For those who want to check the detailed information about the selection process will be directed to an adjacent page, in which the entire history of commentary submissions and minimum descriptions of the reasons of selection are recorded. We believe this open policy will significantly increase the trust for the journal's editorial process.

Thirdy, the journal should adopt transparency policies not only in the PPPR selection, but in its entirety, in order to gain and maintain the trust and reputation from the 
potential contributors. Although $B B S$ is a prestigious journal in the traditional sense, we presume that high prestige is not a necessary requirement to be a good platform of PPPR. In contrast, the transparency criteria, such as those applied to the PPPR section, must be established for the journal as a whole. They could effectively remove suspicions for biased handling of commentaries, and give the audience and potential contributors a high trust for the journal. It is difficult to imagine how PPPR can be maintained without such transparency criteria, so they must be firmly implemented.

Another concern on the incentive for potential contributors is whether they can remain anonymous. Since there have been so many pros and cons to the anonymity issue, this requires a rather detailed argument. In short, the proposed PPPR system will accept both signed and unsigned reviews through the same selection process, but we estimate the majority of the submission would be non-anonymous because of its incentive structure. We will discuss this concern separately in the "Anonymity" section below.

(2) Readability: Create a streamlined system that automatically generates pairings between the target article and PPPR commentaries.

We propose that the PPPR section should stick to the traditional style of academic journals in its appearance, while utilizing the current web technology as much as possible in the background. By referring to traditional styles, we principally suppose the structures of BBS commentary. It must be well paraphrased and organized so that the majority of the audience can grasp the points rapidly and easily. Reference must be completed with relevant information (e.g. in APA style).

Then, our challenge would be how to integrate such an old-fashioned style with the unlimited number of target and commentary articles. We propose to utilize the current open science infrastructure to its full extent for this goal. Firstly, if the preprint of the target article is available on a public repository (e.g. PsyArXiv) with the CC-BY licence, 
its information will be extracted and transplanted to a pre-designated template to create the "root" of target-commentary pairings. If it's not available, this part would be substituted by the title, author information, abstract etc. of the original article, which can be cited from public databases such as PubMed (https://pubmed.ncbi.nlm.nih.gov/).

Secondly, a review commentary that successfully passed the editorial selection will be given a DOI as well as CC-BY licence and re-formatted into a design unified with the target article (or its partial information). High readability must be maintained with a simple and integrated format without any unnecessary information (links to ads, related articles, SNS services etc.), so that the disturbance for the readers will be kept at a minimal level. When the second commentary comes in, it will be added after the first one, creating an ever-growing succession of articles. An index at the header area might be helpful to guide the readers to the related information. A similar idea for the pairing format (but not the full-length quotation) has been already implemented in PREview, for example (https://www.prereview.org/).

Finally, there is another problem caused by the ever-lasting accumulation of commentaries; i.e. what is the best way to organize them in order to maximize their information values. Some PPPRs (e.g. bioRixv, ScienceOpen) adopt rating systems to solve this issue. These systems are similar to the "like/unlike" button on various SNS services, requiring just a single click for rating. The review which has gained the highest rating will be shown at the top of the commentary section and the rest will be ordered accordingly. This is probably the easiest method to reflect the information value of each commentary into the structure of the target-commentary pairings.

\section{Anonymity}

One of the issues concerning PPPR that has been most frequently debated is whether the commenter's anonymity should be protected or not. Apparently, the majority of the 
researchers think that to make reviews as well as the reviewer's identities open to the public is not a good idea. According to a recent survey, $48.6 \%$ of the respondents said that it would be unlikely (or very unlikely) for them to accept the review invitation, if their identities and the content of the review commentaries would be publicized (Publon, 2018). Another research suggested that $73.9 \%$ of the researchers thought that it would be unwise to deprive the right for choosing their anonymity from reviewers (they chose "agree" or "strongly agree" responses to the questionnaire item). They (67.2\%) also expressed unwillingness to contribute to reviewing articles in a journal that adopted such open policies (agree/strongly agree). Finally, $65.2 \%$ answered that they would be more reluctant to write strongly critical reviews under such systems (Taylor \& Francis Group, 2015; see also Ross-Hellauer et al., 2017).

Some speculate that these widely observed attitudes are probably due to the fear among the researchers for the possibility of retaliation by their (superior) colleagues (McCook, 2018; Neuroskeptic, 2015). They advocated anonymity arguing that such fear could wipe out many valuable criticisms, and therefore significantly distort not only the review process but also the entire research field. Others, on the other hand, insisted that anonymity would only make commenters irresponsible, sometimes malicious, or even prone to conflicts of interest. They argued that therefore it should not be regarded as the norm, except the case of whistleblowers (Blatt, 2015; Schneider, 2015).

Certainly, this issue is quite critical to our proposal. Even if we could successfully implement PPPR as designed in the current article, it might be possible that only a few people would dare to submit reviews, knowing that every information would be disclosed. Then, our idea could end up in the same situation as the current other PPPR.

The researchers' assessment on open identity, however, may have another interesting aspect. A report showed that a greater number of researchers $(44 \%)$ agreed (or strongly agreed) that non-anonymity could improve the quality of reviews than those (35\%) who 
disagree (or strongly disagree) (Ross-Hellauer et al., 2017). Likewise, the number of those who thought that disclosing reviewers' name was a fair action to the authors $(44 \%$, agree/strongly-agree) was greater than those not $(30 \%$, disagree/strongly-disagree). Thus, it could be the case that at least a non-negligible portion of researchers are inherently supporting the non-anonymous review system, but the fear of retaliation hinders them from further commitments.

It should be emphasized, however, that we actually do not have any idea how realistic this fear of retaliation is. The majority of researchers and academic journals have never experienced the open-identity, open-report review style, therefore we have no empirical data to estimate the frequency and the amount of damage that the retaliation could possibly have. Furthermore, even if the fear is truly a concern, we still don't know how it will be affected when the number of researchers engaged in the open-review activity increases. We should not downplay the threat without a good reason, but should also admit that we just do not know much about it.

In contrast, as we have already pointed out, there are some legitimate reasons to believe that PPPR could significantly benefit science by improving the precision and effectiveness of the paper evaluation. When this advantage is contrasted with the underdetermined estimate of fear, we see no reason not to give a chance to the new proposal, in the form of a limited-time trial for example. Note also that the data and discussions we mentioned above are all based on the current peer-review system. The additional incentives that the newly proposed PPPR would provide can potentially be a catalyst to tip the trend.

Finally, we also like to emphasize that our appeal for testing the non-anonymous PPPR does not mean that there should be no space for anonymity. A freedom for choosing anonymity must be reserved especially for the case of whistleblowers. We therefore think that the proposed PPPR system should also accept anonymous commentaries 
through the same process as signed contributions. We believe, however, that the lack of incentive could specifically affect and discourage anonymous commenters and therefore their numbers could be significantly suppressed. Given the critical importance they could have to the scientific community, special care must be taken for them not to withdraw into silence. This should be treated separately from the standard review system described in this article, as in the case for the various harassment policies. We will leave this discussion for a different occasion.

\section{Conclusion}

In this paper, we proposed a new PPPR system that might solve the problem of unpopularity that other PPPRs are currently facing. We speculate that the biggest cause of the problem is the lack of strong incentives among potential commenters. We looked at the open peer commentary section of $B B S$ as a reference, and implemented its features in the current web-based, open research environment. As in some other existing PPPR systems, target articles will be taken from any academic media including preprints, and are reviewed by the unlimited number of commentaries. It critically differs from others, however, regarding that the review commentaries will be qualified, if minimally, by journal editors, and published in a section specifically dedicated to PPPR. The section will be designed to achieve high readability, with the relevant information (or entire body) of the target article and review commentaries unified within an integrated template.

In a sense, the style we proposed for the new PPPR inherited many features of the traditional academic publications. The selection and publication processes, as well as writing a well-organized commentary definitely requires much more time than the quick and simple posting of short comments. High readability with a simple text format will sacrifice the richness of information embedded within the web of hypertexts. At the same time, however, such seemingly anachronistic, slow approach could provide a high 
level of trust towards the system, and consequently guarantee the status of the commentaries equivalent to genuine publications. We believe that, although their intentions were undoubtedly correct, previous attempts to establish PPPR might have been too radical and too "information savvy" to gain popularity within an ordinary research community.

Finally, we have emphasized the filtering function of PPPR so far, but it is by no means not excluding the fostering characteristics of peer review. In fact, the BBS commentary section provides us with many good examples of such positive discussions and theory developments. Our proposed PPPR format also can be used in the same way. We believe that frequent and deep conversations between researchers is at the core of scientific progress, and this new publication system could help those activities too, ultimately benefiting science in general. (4387 words)

\section{Reference}

Amsen, E. (2014, July 8). What is post-publication peer review? F1000 Blogs. https://blog.f1000.com/2014/07/08/what-is-post-publication-peer-review/

Armstrong, J. S. (1997). Peer review for journals: Evidence on quality control, fairness, and innovation. Science and Engineering Ethics, 3(1), 63-84. https://doi.org/10.1007/s11948-997-0017-3

Bentley, P. J. (2009). The game of funding: Modelling peer review for research grants.

Proceedings of the 11th Annual Conference Companion on Genetic and Evolutionary Computation Conference: Late Breaking Papers, 2597-2602.

https://doi.org/10.1145/1570256.1570367

Blatt, M. R. (2015). Vigilante Science. Plant Physiology, 169(2), 907-909.

https://doi.org/10.1104/pp.15.01443 
Bornmann, L., Mutz, R., \& Daniel, H.-D. (2010). A Reliability-Generalization Study of Journal Peer Reviews: A Multilevel Meta-Analysis of Inter-Rater Reliability and Its Determinants. PLOS ONE, 5(12), e14331. https://doi.org/10.1371/journal.pone.0014331

Botvinik-Nezer, R., Holzmeister, F., Camerer, C. F., Dreber, A., Huber, J., Johannesson, M., Kirchler, M., Iwanir, R., Mumford, J. A., Adcock, R. A., Avesani, P., Baczkowski, B. M., Bajracharya, A., Bakst, L., Ball, S., Barilari, M., Bault, N., Beaton, D., Beitner, J., ... Schonberg, T. (2020). Variability in the analysis of a single neuroimaging dataset by many teams. Nature, 1-7. https://doi.org/10.1038/s41586-020-2314-9

Bravo, G., Grimaldo, F., López-Iñesta, E., Mehmani, B., \& Squazzoni, F. (2019). The effect of publishing peer review reports on referee behavior in five scholarly journals. Nature Communications, 10(1), 322. https://doi.org/10.1038/s41467-018-08250-2

Brown, C. T. (2016, February 15). The likely challenges of (post) publication peer review. http://ivory.idyll.org/blog/2016-on-pppr.html

Camerer, C. F., Dreber, A., Forsell, E., Ho, T.-H., Huber, J., Johannesson, M., Kirchler, M., Almenberg, J., Altmejd, A., Chan, T., Heikensten, E., Holzmeister, F., Imai, T., Isaksson, S., Nave, G., Pfeiffer, T., Razen, M., \& Wu, H. (2016). Evaluating replicability of laboratory experiments in economics. Science, 351(6280), 1433-1436. https://doi.org/10.1126/science.aaf0918

Camerer, C. F., Dreber, A., Holzmeister, F., Ho, T.-H., Huber, J., Johannesson, M., Kirchler, M., Nave, G., Nosek, B. A., Pfeiffer, T., Altmejd, A., Buttrick, N., Chan, T., Chen, Y., Forsell, E., Gampa, A., Heikensten, E., Hummer, L., Imai, T., ... Wu, H. (2018). Evaluating the replicability of social science experiments in Nature and Science between 2010 and 2015. Nature Human Behaviour, 2(9), 637. https://doi.org/10.1038/s41562-018-0399-z Dolgin, E. (2018). PubMed Commons closes its doors to comments. Nature. 
https://doi.org/10.1038/d41586-018-01591-4

Doyen, S., Klein, O., Pichon, C.-L., \& Cleeremans, A. (2012). Behavioral Priming: It's All in the Mind, but Whose Mind? PLOS ONE, 7(1), e29081.

https://doi.org/10.1371/journal.pone.0029081

Dreber, A., Pfeiffer, T., Almenberg, J., Isaksson, S., Wilson, B., Chen, Y., Nosek, B. A., \& Johannesson, M. (2015). Using prediction markets to estimate the reproducibility of scientific research. PNAS Proceedings of the National Academy of Sciences of the United States of America, 112(50), 15343-15347.

https://doi.org/10.1073/pnas.1516179112

Eisen, M. (2016, January 21). Coupling Pre-Prints and Post-Publication Peer Review for Fast, Cheap, Fair, and Effective Science Publishing. http://www.michaeleisen.org/blog/?p=1820

Everett, J. A. C., \& Earp, B. D. (2015). A tragedy of the (academic) commons: Interpreting the replication crisis in psychology as a social dilemma for early-career researchers. Frontiers in Psychology, 6. https://doi.org/10.3389/fpsyg.2015.01152

Forsell, E., Viganola, D., Pfeiffer, T., Almenberg, J., Wilson, B., Chen, Y., Nosek, B. A., Johannesson, M., \& Dreber, A. (2019). Predicting replication outcomes in the Many Labs 2 study. Journal of Economic Psychology, 75, 102117. https://doi.org/10.1016/j.joep.2018.10.009

Gelman, A. (2016, December 16). An efficiency argument for post-publication review "Statistical Modeling, Causal Inference, and Social Science. https://statmodeling.stat.columbia.edu/2016/12/16/an-efficiency-argument-for-post-public ation-review/

Hagger, M. S., Chatzisarantis, N. L. D., Alberts, H., Anggono, C. O., Batailler, C., Birt, A. R., 
Brand, R., Brandt, M. J., Brewer, G., Bruyneel, S., Calvillo, D. P., Campbell, W. K., Cannon, P. R., Carlucci, M., Carruth, N. P., Cheung, T., Crowell, A., De Ridder, D. T. D., Dewitte, S., ... Zwienenberg, M. (2016). A Multilab Preregistered Replication of the Ego-Depletion Effect. Perspectives on Psychological Science, 11(4), 546-573. https://doi.org/10.1177/1745691616652873

Hopewell, S., Collins, G. S., Boutron, I., Yu, L.-M., Cook, J., Shanyinde, M., Wharton, R., Shamseer, L., \& Altman, D. G. (2014). Impact of peer review on reports of randomised trials published in open peer review journals: Retrospective before and after study. BMJ, 349. https://doi.org/10.1136/bmj.g4145

Jha, A. (2012, September 13). False positives: Fraud and misconduct are threatening scientific research. The Guardian. http://www.theguardian.com/science/2012/sep/13/scientific-research-fraud-bad-practice

Johnston, D. (2015). Peer review incentives: A simple idea to encourage fast and effective peer review. European Science Editing, 2.

Knoepfler, P. (2015). Reviewing post-publication peer review. Trends in Genetics : TIG, 31(5), 221-223. https://doi.org/10.1016/j.tig.2015.03.006

Kriegeskorte, N. (2009, February 8). Open post-publication peer review. The Future of Scientific Publishing. https://futureofscipub.wordpress.com/open-post-publication-peer-review/ Le Texier, T. (2019). Debunking the Stanford Prison Experiment. American Psychologist, 74(7), 823-839. https://doi.org/10.1037/amp0000401

Lindsey, D. (1988). Assessing precision in the manuscript review process: A little better than a dice roll. Scientometrics, 14(1), 75-82. https://doi.org/10.1007/BF02020243

McCook, A. A. (2018, February 2). PubMed shuts down its comments feature, PubMed Commons. Retraction Watch. 
https://retractionwatch.com/2018/02/02/pubmed-shuts-comments-feature-pubmed-comm ons/

Meskus, M., Marelli, L., \& D’Agostino, G. (2018). Research Misconduct in the Age of Open Science: The Case of STAP Stem Cells. Science as Culture, 27(1), 1-23. https://doi.org/10.1080/09505431.2017.1316975

Neff, B. D., \& Olden, J. D. (2006). Is Peer Review a Game of Chance? BioScience, 56(4), 333-340. https://doi.org/10.1641/0006-3568(2006)56[333:IPRAGO]2.0.CO;2

Neuroskeptic. (2015, December 13). PubPeer and Anonymity in Science. Discover Magazine. https://www.discovermagazine.com/the-sciences/pubpeer-and-anonymity-in-science

Nielsen, M. (2009). Doing science in the open. Physics World, 22(05), 30-35. https://doi.org/10.1088/2058-7058/22/05/38

Open Science Collaboration. (2015). Estimating the reproducibility of psychological science. Science, 349(6251), aac4716-aac4716. https://doi.org/10.1126/science.aac4716

Perry, G. (2012). Behind the Shock Machine: The untold story of the notorious Milgram psychology experiments. Scribe.

Publon. (2018). Global State of Peer Review. http://publons.com/community/gspr

Rohrer, J. M., Egloff, B., \& Schmukle, S. C. (2017). Probing Birth-Order Effects on Narrow Traits Using Specification-Curve Analysis. Psychological Science, 28(12), 1821-1832. https://doi.org/10.1177/0956797617723726

Ross-Hellauer, T., Deppe, A., \& Schmidt, B. (2017). Survey on open peer review: Attitudes and experience amongst editors, authors and reviewers. PLOS ONE, 12(12), e0189311. https://doi.org/10.1371/journal.pone.0189311

Schneider, L. (2015, December 13). Post-publication peer review: Signed or anonymous? For Better Science. 
https://forbetterscience.com/2015/12/13/post-publication-peer-review-signed-or-anonym ous/

Sullivan, B. (2018, August 28). Is It Time for Pre-Publication Peer Review to Die? | PLOS SciComm.

https://scicomm.plos.org/2018/08/28/is-it-time-for-pre-publication-peer-review-to-die/ Taylor \& Francis Group. (2015). Peer review | a global view. Author Services. https://authorservices.taylorandfrancis.com/peer-review/peer-review-global-view/ Tennant, J. (2017, April 12). What are the barriers to post-publication peer review? Impact of Social Sciences.

https://blogs.Ise.ac.uk/impactofsocialsciences/2017/04/12/what-are-the-barriers-to-post-p ublication-peer-review/

van der Zee, T., Anaya, J., \& Brown, N. J. L. (2017). Statistical heartburn: An attempt to digest four pizza publications from the Cornell Food and Brand Lab. BMC Nutrition, 3(1), 54. https://doi.org/10.1186/s40795-017-0167-x

Van Noorden, R. (2014, March 14). The new dilemma of online peer review: Too many places to post? : News blog. http://blogs.nature.com/news/2014/03/the-new-dilemma-of-online-peer-review-too-manyplaces-to-post.html

Wagenmakers, E.-J., Beek, T., Dijkhoff, L., Gronau, Q. F., Acosta, A., Adams, R. B., Albohn, D. N., Allard, E. S., Benning, S. D., Blouin-Hudon, E.-M., Bulnes, L. C., Caldwell, T. L., Calin-Jageman, R. J., Capaldi, C. A., Carfagno, N. S., Chasten, K. T., Cleeremans, A., Connell, L., DeCicco, J. M., ... Zwaan, R. A. (2016). Registered Replication Report: Strack, Martin, \& Stepper (1988). Perspectives on Psychological Science, 11(6), 917-928. https://doi.org/10.1177/1745691616674458 